\title{
Developing and Enhancing Adherence to a Telehealth ABA Parent Training Curriculum for Caregivers of Children with Autism
}

\author{
Zhihui $\mathrm{Yi}^{1} \cdot$ Mark R. Dixon ${ }^{1}$ \\ Published online: 3 November 2020 \\ (C) Association for Behavior Analysis International 2020
}

\begin{abstract}
The current COVID-19 pandemic poses unique challenges to the delivery of applied behavior analysis (ABA) services, and there has been a growing demand for evidence-based practices on how to develop a telehealth ABA service model. The current article provides a detailed technological guide on how to develop a telehealth ABA parent training curriculum. Our model also includes a series of brief acceptance and commitment training (ACT) protocols to enhance parental adherence. We provide the details for replicating our telehealth model and also demonstrate its effectiveness. To begin, a step-by-step guide on how to develop this curriculum is presented, as well as protocols used in a 60-day telehealth ABA parent training program. Afterward, we describe a randomized controlled trial design to examine the effectiveness of this protocol. Thirteen families from the southern Illinois region participated in the curriculum before the COVID-19 outbreak. Obtained data indicated training was effective to teach skills, and parents with supplemental ACT material made significantly more progress than those in the control group, $t(11)=$ $2.36, p=.038$. Halfway through the training, the outbreak of COVID-19 occurred, and parents in the ACT group were more likely to continue the program, whereas parents in the control group were significantly more likely to postpone their participation, $\chi^{2}=6.96, p=.008$. Social validity measures indicated that parents rated the curriculum favorably. Limitations and suggestions for practitioners are discussed.
\end{abstract}

Keywords ABA $\cdot$ ACT $\cdot$ Parent training $\cdot$ Parental adherence $\cdot$ Telehealth

The recent pandemic of COVID-19 in the United States has caused significant disruption to people's daily lives. As of April 14, 2020, there have been approximately 200,000

Portions of this research was funded by the State of Illinois's The Autism Program (TAP) awarded to the second author. Research was conducted at the Southern Illinois University.

Editor's Note

This manuscript is being published on a highly expedited basis, as part of a series of emergency publications designed to help practitioners of applied behavior analysis take immediate action to adjust to and mitigate the COVID-19 crisis. This article was submitted on April 14, 2020, and received final acceptance on April 22, 2020. The journal would like to especially thank Dr. Amanda Kelly for her expeditious review of the manuscript. The views and strategies suggested by the articles in this series do not represent the positions of the Association for Behavior Analysis International or Springer Nature.

Mark R. Dixon mrdixon@uic.edu

1 Department of Disability and Human Development, College of Applied Health Science, University of Illinois at Chicago, Chicago, IL 60608, USA confirmed cases of COVID-19 in the United States (Centers for Disease Control and Prevention, 2020). Forty-four states, including the District of Columbia, have issued stay-at-home orders, limiting travel to essential activities only, and closing all nonessential businesses. Although services related to autism treatment are considered essential services, practitioners have voiced concerns on the risk of continuing in-person services that require the therapist and client to remain in physical proximity, such as applied behavior analysis (ABA), occupational therapy, and physical therapy. In a recent article, Cox, Plavnick, and Brodhead (2020) argued against a blanket interpretation of in-person ABA services as essential because providing these services may present significant risks to clients and their families. The authors also proposed a case-by-case decision framework and advocated for the transition to telehealth or other remote service modalities when possible. Additional articles that have been appearing in Behavior Analysis in Practice have advocated for the use of digital activity schedules (Reinert, Higbee, \& Nix, 2020), the adaption of programs for telehealth services (Rodriguez, 2020), and functional communication trainings (Bondy, Horton, \& 
Frost, 2020). These installments provide excellent summaries and recommendations that are consumable for the practicing behavior analyst, and additional work that documents the successes of these approaches within the world of COVID-19 would be welcomed in the literature.

The field of ABA has seen a rapid increase in interest in providing services remotely. Numerous organizations have published guidelines on how to continue ABA services using a telehealth model during the COVID-19 pandemic. For example, the Council of Autism Service Providers (CASP; 2020) issued guidelines on various telehealth service delivery models, claiming reimbursements, and service-specific guidance. Similarly, the Behavior Analyst Certification Board (BACB, 2020) has also issued ethical guidelines for ABA providers during these times. These national organizations, along with regional ABA associations, have also hosted a variety of webinars to facilitate the transition to an ABA telehealth model. However, most agencies are unfamiliar with the process of developing an ABA telehealth program from scratch, and there are a limited number of publications investigating different parameters in ABA telehealth models. Tomlinson, Gore, and McGill (2018) conducted an extensive literature review on this topic and identified 20 studies that utilized a telehealth model. Cumulatively, their findings suggested promising outcomes, as trainees were able to implement various behavior-analytic techniques (e.g., conduct preference assessments and functional analysis, implement differential reinforcement techniques, provide discrete-trial teaching) following the telehealth intervention (Fischer et al., 2016; Hay-Hansson \& Eldevik, 2013; Higgins, Luczynski, Carroll, Fisher, \& Mudford, 2017; Lindgren et al., 2016). However, only 3 of the 20 studies involved the participation of the client's parents, and all of the telehealth models focused on specific clinical skills rather than overall behavioral principles. Therefore, these models pose a unique challenge under the current COVID-19 situation, as parents, in most cases, are now required to work from home and do not necessarily have the time, skills, or resources to provide direct intervention consistently. Furthermore, it is not clear if a crisis like COVID-19 would impact the adherence to telehealth protocols, and if such adherence could be improved upon in any objective way.

To address this challenge, an alternative is to provide ondemand training to parents on general behavior-analytic principles, focusing the training on teaching basic skills and behavioral management techniques that can be completed at their own pace, and to evaluate its durability to withstand attrition during the COVID-19 pandemic. This model could also include components to enhance parental adherence and generalization of the skills taught so parents can easily apply them at home. Most importantly, the model should allow for flexibility so that parents can receive training on issues of significance to them. Thus, the purpose of the current article is to provide guidance on the techniques needed for developing such a program. The rationale and evidence that support the various components of our current parent program, such as risk assessment systems (Brodhead, 2015; McIntyre, Gresham, DiGennaro, \& Reed, 2007), competence-based contingencies (Allen \& Warzak, 2000; Loeber \& Weisman, 1975), use of non-behavior-analytic terms (Becirevic, Critchfield, \& Reed, 2016), and acceptance and commitment therapy to increase parental adherence (Gould, Tarbox, \& Coyne, 2018), have all been vetted within the behavioranalytic literature. In this installment, we focus on providing behavior analysts with a step-by-step guide to directly replicate this program in the era of COVID-19. We also document that this program does indeed work, and how it can be enhanced in terms of parent adherence to interventions.

\section{Study 1: Description of the Yi and Dixon On-Demand Telehealth Model}

\section{Program Outline}

Activities and lessons in the program are designed so the parent can complete the program within 60 calendar days. Our telehealth ABA parent training curriculum consists of the following four components:

1. An onboarding meeting. A group onboarding meeting is first conducted to provide an overview of the program. The meeting is hosted by a clinical supervisor and includes a brief introduction to the protocol, as well as providing technological coaching to ensure that parents can successfully use the software and platforms required for the program.

2. Five self-paced online skill development lessons. All five lessons are created and hosted via an online learner management platform called (Teachable 2020) for parents to complete in their own time. The platform we chose is not critical for the instructional design, and alternatives such as Desire2Learn (2020) and Moodle (2020) would be functionally equivalent. Each lesson covers several topics on basic behavior-analytic principles, skill teaching, and behavior management techniques (see the online training lessons that follow for detail). Each lesson is further divided into several modules. At the end of each module, there is a brief knowledge check. The knowledge check includes three to five multiple-choice questions. There are guided activities throughout the module to help parents adapt the lesson content into techniques they can use at home. At the end of each online lesson, there is an activity in which parents are required to record themselves using one technique they learned in the lesson. 
3. Five individual consultations with follow-up coaching. Upon completing each lesson, and demonstrating adequate proficiency on quizzes, parents meet with a Board Certified Behavior Analyst (BCBA) or a senior clinician to receive individual live consultation throughout the program. Each of the five separate consultation sessions lasts $45 \mathrm{~min}$, and each consultation is structured in relation to one online lesson (see the individual consultation that follows for detail). During the last consultation meeting, parents receive a behavior intervention plan they can implement at home. Afterward, parents receive weekly check-ins from the treatment team for additional coaching.

4. Competency-based contingency. Parents are required to complete one online lesson before they can schedule a meeting with the treatment team. Before scheduling the first meeting, the treatment team verifies that the parent has completed the first online lesson, which also includes required activities. A workbook is provided to each family, and parents are required to use the workbook to finish those activities. Parents are also required to submit the video in which they recorded themselves implementing techniques learned during the online skill development lessons. Once the treatment team verifies that all those conditions have been met, they reach out to the family to schedule one online consultation meeting. After the meeting, parents need to complete the next online lesson, along with all the activities, before they can schedule the second consultation. This continues for the third, fourth, and fifth online lessons/consultations. Once parents finish all five lessons, the treatment team provides them with the behavior intervention plan, and they begin receiving weekly check-ins. Figure 1 provides a flowchart that describes this process.

\section{Risk Analysis}

Before onboarding, the treatment team first conducts a risk analysis to determine whether there are immediate risks caused by maladaptive behavior. Clients are assigned to one of four tiers based on the history and complexity of their maladaptive behaviors. Clients assigned to Tier 1 demonstrate minimum maladaptive behavior. For these clients, the treatment team develops a behavior intervention plan for one common problem behavior. The family receives one 30 -min phone call or meeting per week during the follow-up coaching. Clients assigned to Tier 2 demonstrate some maladaptive behavior. Although these behaviors are more frequent, they do not pose immediate risks and can often be redirected. These families receive behavior plans for two or three maladaptive behaviors and receive two 30-min phone

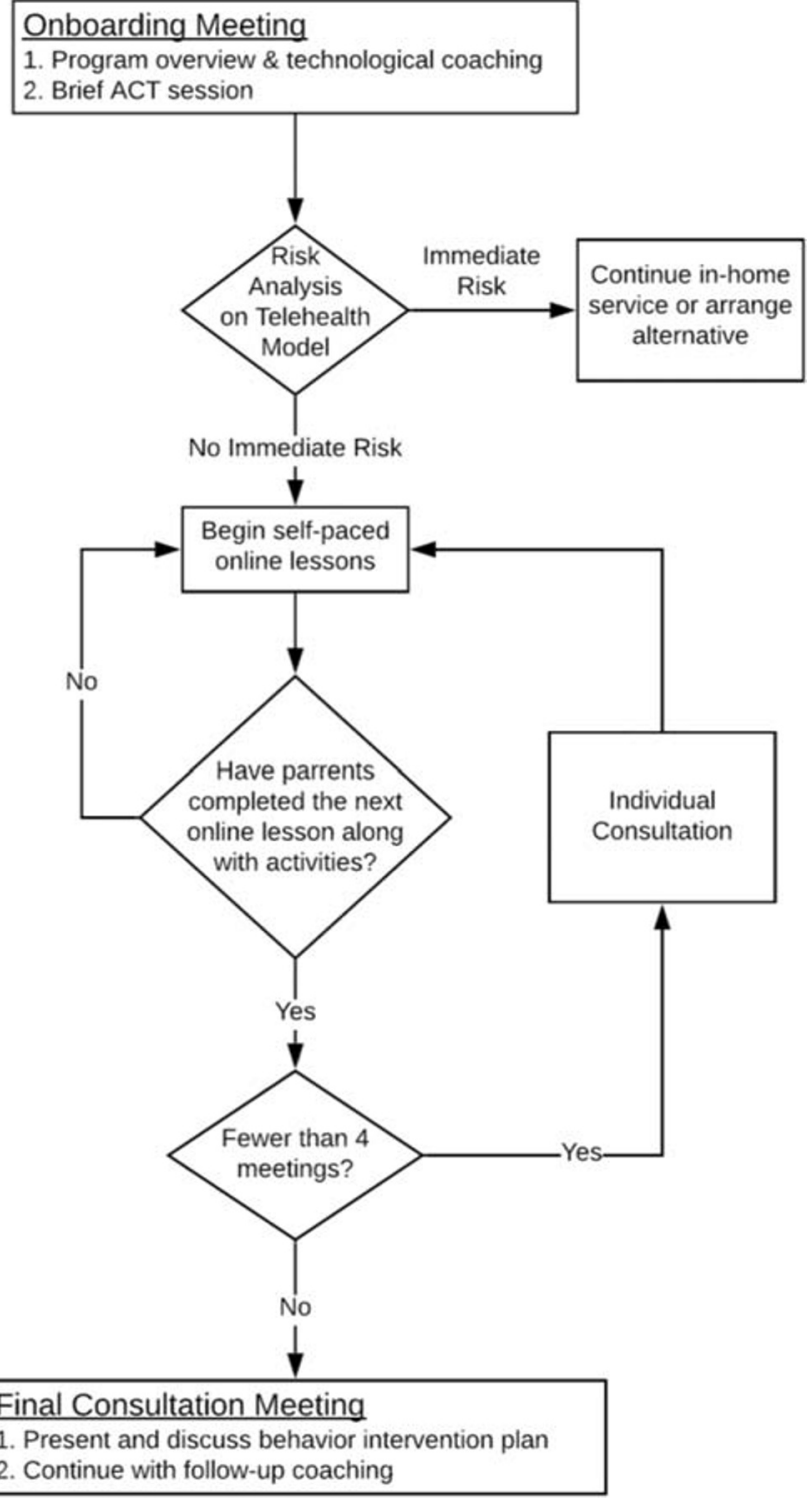

Fig. 1 Flowchart for telehealth ABA parent training curriculum

calls or meetings per week during the follow-up coaching. Clients assigned to Tier 3 demonstrate several maladaptive behaviors, and these behaviors are consistent across multiple settings. However, these behaviors do not pose immediate risks (e.g., no self-injurious behavior or severe property destruction). These families receive behavior plans for two maladaptive behaviors and receive two 30-min phone calls in two settings (e.g., in the home and in community outings). Clients assigned to Tier 4 demonstrate severe maladaptive behaviors that pose immediate risks. These families continue to receive in-home services or receive crisis management services.

\section{Onboarding}

Program overview and technological coaching During the program overview, the clinical supervisor explains the structure of 
the program to the parent. The clinical supervisor briefly presents the content in each online lesson and explains the requirement of completing the online lesson and activities before scheduling individual consultations. The clinical supervisor also demonstrates how to use the online platform and provides examples of different types of activities in online lessons. The clinical supervisor then provides brief technological coaching on the online meeting software (e.g., Zoom, GoToMeeting). Specifically, the supervisor explains (a) how to find the link to join the meeting; (b) what material to prepare before the meeting; (c) how to configure the computer, the webcam, and the microphone in the meeting software; and (d) how to share files, videos, or the computer screen during the meeting. Finally, a workbook is given to the parent, along with a prerecorded video on the technological tutorial. The technological tutorial video covers the same content as in the coaching session on the online meeting software so parents can reference the video as needed throughout the curriculum.

Brief acceptance and commitment training (ACT) session During the onboarding meeting, the clinical supervisor conducts a brief ACT session. The ACT session lasts about $30 \mathrm{~min}$ and is delivered to parents and caregivers in a group setting. For each activity, the clinician reads from a script to facilitate the guided group activity. These activities aim to increase parents' present moment awareness, clarify their values, and promote value-driven behaviors. Table 1 summarizes the protocol used in the ACT session. The activities are adapted from the ACT toolkit organized by Ahles and Jenkins (2018). The page number to the right of the activity indicates where a detailed description and materials needed can be found within the toolkit. After these five activities, the clinician concludes the onboarding meeting. The ACT component in this model also includes a weekly text message, which will be discussed in the progress monitoring portion of the model.

\section{Online Training Lessons}

There are five self-paced online lessons in this program. Parents are required to complete these lessons in sequential order. Each lesson is further divided into different modules. At the end of each module, there is a brief knowledge check, each

Table 1. Protocol Used in the ACT Session

\begin{tabular}{|c|c|c|c|}
\hline Order & Activity & Page & Description \\
\hline 1 & Guided meditation: mindful breathing & 32 & $\begin{array}{l}\text { The first activity presented to parents is a guided meditation that focuses on mindful } \\
\text { breathing. The clinician begins by asking parents to close their eyes and sit comfortably, } \\
\text { bringing their attention to their breathing. The clinician then asks parents to imagine having } \\
\text { a balloon in their stomach and, as they breathe in and out, to pay attention to how the } \\
\text { balloon would inflate and deflate. Parents are instructed that if at any point they notice } \\
\text { thoughts coming into their mind, they should acknowledge those thoughts and direct their } \\
\text { attention back to their breath. As the exercise concludes, the clinician will then ask parents } \\
\text { to slowly open their eyes. This exercise lasts about } 3 \text { min. }\end{array}$ \\
\hline 2 & $\begin{array}{l}\text { Experiential exercise: "Eating One Raisin: A First } \\
\text { Taste of Mindfulness" }\end{array}$ & 31 & $\begin{array}{l}\text { The clinician facilitates an experiential activity titled "Eating One Raisin: A First Taste of } \\
\text { Mindfulness" and focuses on present moment awareness. The clinician asks each parent to } \\
\text { put a few raisins on a napkin before beginning the activity. The clinician then directs the } \\
\text { parents' attention to the raisin and guides them to observe, touch, smell, taste, and } \\
\text { eventually swallow the raisin. During each step, the clinician prompts parents to pay close } \\
\text { attention to the different sensory inputs. The activity concludes by the clinician asking } \\
\text { parents to notice how their bodies felt as they completed the activity. This exercise takes } \\
\text { approximately } 5 \text { min to complete. }\end{array}$ \\
\hline 3 & Experiential exercise: "Five Senses" & 34 & $\begin{array}{l}\text { The clinician leads an experiential activity titled "Five Senses." This activity also targets } \\
\text { present moment awareness. The activity begins with the clinician asking parents to look } \\
\text { around the room and notice five things they have not noticed before. The clinician then } \\
\text { directs parents to attend to their other senses, asking them to identify something they feel, } \\
\text { hear, smell, and taste. The clinician asks the parents to briefly share with the group one of } \\
\text { the new things that they have not noticed before. This exercise takes } 5 \text { min to complete. }\end{array}$ \\
\hline 4 & Experiential exercise: "Facing Your Feelings" & 15 & $\begin{array}{l}\text { Parents complete an experiential exercise titled "Facing Your Feelings." This activity is a } \\
\text { guided meditation that aims to teach participants to distance themselves from their thoughts } \\
\text { and feelings. The clinician first asks parents to close their eyes and direct their attention to } \\
\text { their emotions in the present moment during the meditation. The clinician will frequently } \\
\text { redirect parents to pay attention to their breathing and discuss how emotions and feelings } \\
\text { come and go. This exercise takes about } 5 \text { min to complete. }\end{array}$ \\
\hline 5 & $\begin{array}{l}\text { Reflect on your values; } 10 \text { tips for motivating } \\
\text { yourself to practice new skills or pursue } \\
\text { important goals }\end{array}$ & $\begin{array}{l}41 \\
45-47\end{array}$ & $\begin{array}{l}\text { The last activity focuses on values and value-driven behaviors. The clinician first presents a } \\
\text { list with many different values and asks the parents to circle the values that are the most } \\
\text { important to them. Once they select their most important values, the clinician reviews } 10 \\
\text { steps they can take to incorporate their values into actions within their daily lives. The } \\
\text { clinician then asks parents to write down those thoughts and ideas and to share their } \\
\text { thoughts briefly. This activity takes about } 10 \text { min to complete. }\end{array}$ \\
\hline
\end{tabular}


containing three to five multiple-choice and/or true-or-false questions. Throughout the module, there are various activities. Parents are required to use the workbook to write down their answers to these activities. See Figure 2 for an example. At the end of each lesson, there is one cumulative activity named "Do-It-Yourself," where parents are required to record themselves using one technique they just learned in the lesson. Table 2 summarizes the contents covered in each lesson, along with the required activities. Note that the course content outlined there should be used as a reference, and clinicians should make case-by-case decisions as to whether the content is appropriate.

Preparing course material When preparing course materials, clinicians need to pay attention to avoid using certain behavior-analytic terms, as they can be technical and confusing. For example, instead of using the term "Premack Principles," clinicians can use "Grandma's Rule." Instead of using the term "prompt," clinicians can use "give hints during teaching." Instead of using the terms "tangible," "escape," "attention," and "automatic" to describe the function of behavior, clinicians can use "behave to get something," "behave to get away from something," "behave to get attention," and "behave because it just feels good." See Figure 3 for examples of slides explaining functions of behavior.

The clinician should also prepare abundant examples and scenarios so parents can easily understand the course content. For example, Lesson 1 mentions that the same behavior in different contexts could cause different outcomes, and people may define the same behavior differently. It then gives the following scenario:

For example, you are sitting in your car listening to music while you are stopped at a traffic light. If the traffic light is red, everyone behind you will wait patiently because you're following the law. If the traffic light is green, people behind you will get mad and honk their horns, and you might just cause a small traffic jam. As you can see, although it is the same behavior, under different circumstances, people respond to it differently. From your perspective, you didn't notice the light
Fig. 2 Example of the workbook

\section{Lesson 1: What is Behavior?}

Module Two: Why Does Behavior Occur?

Activity: Consider the following scenario and answer the questions below.

It was a Sunday night and the family just finished eating dinner. After dinner Dad asked Tom to finish his chores. Tom did so and Dad gave him 5 dollars.

What is the antecedent?

What is the consequence?

Is the consequence a reinforcer or a punisher?

Activity: What is the potential function of the following behaviors?

1. Taylor engages in rocking behavior (rocking back and forth) when her teacher removes the toys she is playing with.

Possible function:

2. John engages in rocking behavior when his teacher is working with other children. Possible function:

3. Sally engages in rocking behavior while sitting at her desk. Possible function:

4. Mikey engages in rocking behavior when it is time to exercise in P.E. class.

Possible function:

\section{Do-It-Yourself!}

What behavior did you record?

What happened before the behavior? 
Table 2. Contents Covered in Each Lesson and Related Activities

\begin{tabular}{|c|c|c|c|c|}
\hline Lesson & Module & Content & Activities & Do-It-Yourself \\
\hline \multirow[t]{2}{*}{$\begin{array}{l}\text { Lesson 1: What Is } \\
\text { Behavior? }\end{array}$} & $\begin{array}{l}\text { Module 1: What Is } \\
\text { Behavior? }\end{array}$ & $\begin{array}{l}\text { 1. Different people describe behavior } \\
\text { differently; } \\
\text { 2. Behavior and its relationship to } \\
\text { context; } \\
\text { 3. How to define behavior; and } \\
\text { 4. Record behavior via frequency, } \\
\text { duration, and video. }\end{array}$ & $\begin{array}{l}\text { Watch a brief video and come up with } \\
\text { the definition of behavior. }\end{array}$ & $\begin{array}{l}\text { Select one behavior of the child and use } \\
\text { a cell phone to record a short video. } \\
\text { Write down what happens before } \\
\text { and after that behavior. Write down } \\
\text { its potential function. }\end{array}$ \\
\hline & $\begin{array}{c}\text { Module 2: Why Does } \\
\text { Behavior Occur? }\end{array}$ & $\begin{array}{l}\text { 1. Antecedents and consequences of } \\
\text { behavior; } \\
\text { 2. Reinforcer and punisher; } \\
\text { 3. The function of behavior; and } \\
\text { 4. How to determine the potential } \\
\text { function of the behavior. }\end{array}$ & $\begin{array}{l}\text { 1. Identify the antecedent and } \\
\text { consequence in the given scenario, } \\
\text { and identify whether the } \\
\text { consequence is a reinforcer or a } \\
\text { punisher. } \\
\text { 2. Identify the potential function of } \\
\text { behaviors. }\end{array}$ & \\
\hline \multirow[t]{3}{*}{$\begin{array}{l}\text { Lesson 2: Application to } \\
\text { Behavior Management } \\
\text { Part } 1\end{array}$} & $\begin{array}{l}\text { Module 1: Activity } \\
\text { Schedules }\end{array}$ & $\begin{array}{l}\text { 1. What an activity schedule is; } \\
\text { 2. How to make an activity schedule; } \\
\text { and } \\
\text { 3. How to guide the child through an } \\
\text { activity schedule. }\end{array}$ & $\begin{array}{l}\text { Think of a routine that the child } \\
\text { struggles with. Write down each } \\
\text { step of the routine along with what } \\
\text { difficulties the child faces and how } \\
\text { the parent usually responds. }\end{array}$ & $\begin{array}{l}\text { Have parents record themselves using } \\
\text { Grandma's Rule to deliver three } \\
\text { instructions to the child, while } \\
\text { following the guidelines on how to } \\
\text { deliver instructions effectively. }\end{array}$ \\
\hline & $\begin{array}{l}\text { Module 2: } \\
\text { Establishing Rules }\end{array}$ & $\begin{array}{l}\text { 1. How to deliver instructions } \\
\text { effectively; } \\
\text { 2. Notice the child's good behavior; } \\
\text { 3. Follow through with demands and } \\
\text { use a directive tone; and } \\
\text { 4. Use Grandma's Rule (Premack } \\
\text { Principles). }\end{array}$ & $\begin{array}{l}\text { 1. Write down three directions the } \\
\text { parent gave the child today and } \\
\text { identify if it is a positive or negative } \\
\text { statement. } \\
\text { 2. Write down three situations where } \\
\text { the parent can use the Premack } \\
\text { Principles. }\end{array}$ & \\
\hline & $\begin{array}{l}\text { Module 3: Setting Up } \\
\text { the Environment }\end{array}$ & $\begin{array}{l}\text { How to gain instructional control } \\
\text { (content adapted from Seven Steps } \\
\text { for Gaining Instructional Control } \\
\text { by Schramm \& Miller, 2014) }\end{array}$ & $\begin{array}{l}\text { Select one step and write down two } \\
\text { examples of how the parent can use } \\
\text { this in the child's daily routine. }\end{array}$ & \\
\hline \multirow[t]{3}{*}{$\begin{array}{l}\text { Lesson 3: Application to } \\
\text { Behavior Management } \\
\text { Part II }\end{array}$} & $\begin{array}{l}\text { Module 1: Token } \\
\text { Systems }\end{array}$ & $\begin{array}{l}\text { 1. What a token system is; } \\
\text { 2. How to select appropriate tokens; } \\
\text { 3. How to create the price menu or } \\
\text { treasure box; and } \\
\text { 4. How to create a token board. }\end{array}$ & $\begin{array}{l}\text { Identify some items that the child is } \\
\text { willing to work for. Organize them } \\
\text { into small, medium, and large } \\
\text { magnitudes. }\end{array}$ & $\begin{array}{l}\text { Identify a situation where the child } \\
\text { struggles and have parents record } \\
\text { themselves using one technique } \\
\text { covered in the lesson to redirect the } \\
\text { child. }\end{array}$ \\
\hline & $\begin{array}{l}\text { Module 2: Effective } \\
\text { Instruction } \\
\text { Delivery }\end{array}$ & $\begin{array}{l}\text { 1. Get the child's attention; } \\
\text { 2. Use a directive tone of voice; } \\
\text { 3. Be specific in instructions and tell } \\
\text { the child what he or she should do; } \\
\text { and } \\
\text { 4. Follow through. }\end{array}$ & $\begin{array}{l}\text { Identify if the given statements use a } \\
\text { directive tone. }\end{array}$ & \\
\hline & Module 3: Redirection & $\begin{array}{l}\text { 1. What redirection is and when to } \\
\text { redirect; } \\
\text { 2. Use verbal and physical cues to } \\
\text { redirect; } \\
\text { 3. Identify appropriate alternatives } \\
\text { that are functionally equivalent; } \\
\text { and } \\
\text { 4. Use behavior momentum to } \\
\text { redirect. }\end{array}$ & Not applicable & \\
\hline \multirow[t]{2}{*}{$\begin{array}{l}\text { Lesson 4: Teaching New } \\
\quad \text { Skills }\end{array}$} & $\begin{array}{l}\text { Module 1: Natural } \\
\text { Teaching }\end{array}$ & $\begin{array}{l}\text { 1. Use the child's daily routine; } \\
\text { 2. Follow the child's lead; } \\
\text { 3. Get the child's attention; } \\
\text { 4. Reward good behavior; } \\
\text { 5. Reward for trying hard; and } \\
\text { 6. Give hints (prompts) to your child: } \\
\text { vocal hint (verbal prompt), visual } \\
\text { hint (visual prompt), and physical } \\
\text { hint (physical prompt). }\end{array}$ & $\begin{array}{l}\text { 1. Observe the child for } 5 \text { min and } \\
\text { write down what items and } \\
\text { activities the child shows interest } \\
\text { in. Write down how the parent can } \\
\text { use them to motivate the child. } \\
\text { 2. Write down a skill the parent can } \\
\text { teach with that item and the } \\
\text { consequence the parent will } \\
\text { provide. } \\
\text { 3. Think about the child's current skill } \\
\text { level and write down what is a } \\
\text { reasonable attempt. } \\
\text { 4. Think about a skill the child is } \\
\text { learning and write down what type } \\
\text { of hint is most appropriate to use. }\end{array}$ & $\begin{array}{l}\text { Use the material developed in previous } \\
\text { activities and have parents record } \\
\text { themselves conducting a brief } \\
\text { teaching session (5-10 min) with } \\
\text { the child. }\end{array}$ \\
\hline & $\begin{array}{l}\text { Module 2: Structured } \\
\text { Teaching }\end{array}$ & $\begin{array}{l}\text { 1. Characteristics of structured } \\
\text { teaching (discrete-trial teaching); } \\
\text { 2. Setting up the environment before } \\
\text { teaching; } \\
\text { 3. How to present the instruction; } \\
\text { 4. How to reward hard work; } \\
\text { 5. How to keep track of the child's } \\
\text { progress; and }\end{array}$ & $\begin{array}{l}\text { Write down three snack items and } \\
\text { three toys that the child enjoys and } \\
\text { that are also appropriate to use } \\
\text { during structured teaching. }\end{array}$ & \\
\hline
\end{tabular}


Table 2. (continued)

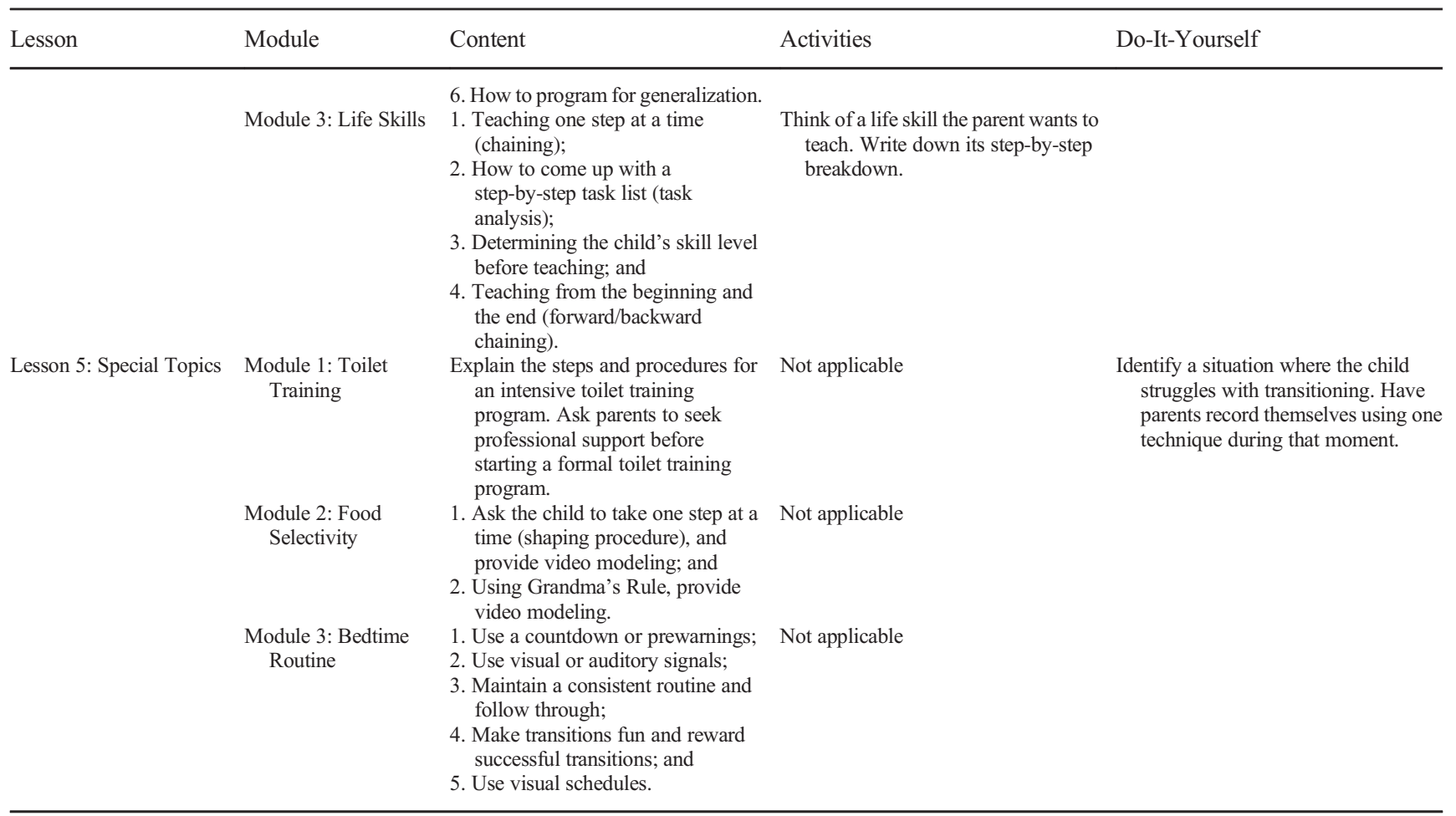

change because you got carried away by the music. You were just not paying attention to the traffic light. The person behind you, however, who just woke up $10 \mathrm{mi}-$ nutes ago, rushing to work because he's about to be late, thought you did that because you were looking at your phone. He becomes really angry since he's already shown up late 2 days in a row. You can see how easy it is for different perspectives to lead to different accounts of the same behavior. This is why defining behavior is really important, so we can be on the same page and know what everyone else is talking about.

When demonstrating specific techniques (e.g., redirection, prompting), clinicians can prepare videos to model the skill. For example, Lesson 4 discusses various prompting techniques that parents can use during teaching. After providing the definition and examples for each type of prompt, a video is shown to the parent where a clinician models how to deliver the prompt. Clinicians can also use a longer video to demonstrate a complex behavioral principle. For example, when the clinician teaches how to conduct structured teaching, parents were educated on the following three areas: how to set up the environment, how to deliver the discriminative stimulus, and how to deliver the reinforcer. Although this seems intimidating, the clinician can first present a video that contains all of these components and ask parents to watch the entire video before diving into those three areas. As the clinician explains what parents need to do in each area, the clinician can represent clips from the same video, pausing and highlighting how the video is in line with the course content they just went through.

Besides preparing examples and video modeling, the clinician should also prepare abundant visual aids to increase userfriendliness and to help highlight important information. For example, clinicians can present different pictures of completed activity schedules and token boards in Lessons 2 and 3.

Format and delivery Once the course content is finalized and the material has been prepared, the clinician can begin assembling the online lesson in its final form. The following steps are suggested as the optimal workflow:

1. Prepare the text content. The first step is to prepare the text to be displayed on each page. Because the deliverable also serves as a handout that parents can refer to, these texts should be complete sentences, rather than bullet points. When preparing the content, also consider how the page is divided. Be concise, and limit the text to no more than 150 words per page.

2. Develop the layout using Microsoft PowerPoint. Once the text content is ready, start putting materials together using Microsoft PowerPoint. First, select a layout that is appropriate for the content. Then, copy and paste the text onto the slide and insert the picture prepared earlier. Use the 


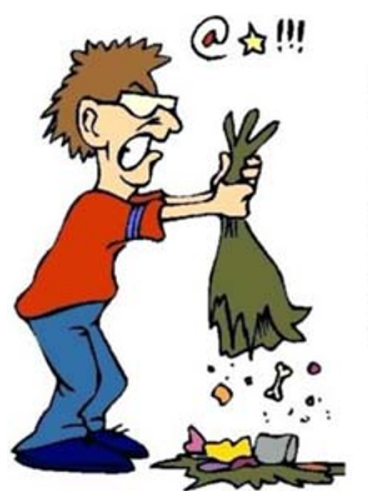

\section{Behave to Get Away from} Something

We engage in this type of behavior so we can get away from something unpleasant.

For example, Bob doesn't like to do chores. Whenever Dad tells Bob to do his chores, Bob hits his younger

brother and Dad always send him to his room. Although Bob gets a time out, he no longer needs to do his chores.

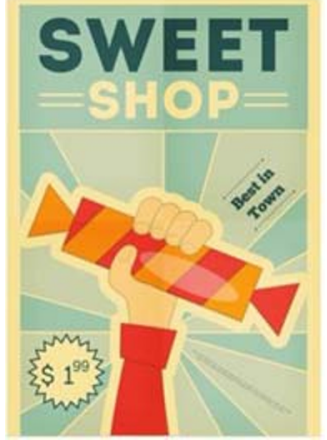

\section{Behave to Get Something}

We engage in this type of behavior to get something we want.

For example, when the child sees candy in the mall, they reach out for it. The function of their behavior is to get that candy.

Fig. 3 Examples of slides explaining the functions of behavior

"Design Ideas" function in PowerPoint to browse automatically generated layouts. Make changes as necessary. The font size for each page's title should range between 28 and 32. The font size for the content should range between 18 and 22. See Figure 4 for examples of finished PowerPoint slides.

3. Prepare the voice-over script. Once the slide is ready, begin preparing the script for the voice-over narrative. This is the script that you will read out loud as you guide parents through the online lesson. This script should be very similar to the content on the slide to avoid confusion. You can add a few sentences to smooth the transition between slides. However, all important information should appear in writing on the slide.

4. Record audio files. Once the voice-over script is ready, begin recording the audio file. Place the microphone in front of your chin. Record one file for each slide of the presentation. Once you hit the record button, wait for 3 s before beginning to speak. Read out the voice-over script with an appropriate volume and speed. Once you finish reading the script, wait for $3 \mathrm{~s}$ before stopping the recording. Name the file appropriately to avoid confusion.

5. Combine the PowerPoint slides with the audio recordings. Before combining the files, first export all PowerPoint slides as JPEG pictures. Open the PowerPoint file with Microsoft PowerPoint, select "File," and then "Export." Change the file format to JPEG. Make sure the box before "Save Every Slide" is checked. Type 1600 in the box after

\section{Behave to Get Attention}

We engage in this type of behavior to get someone's attention.

For example, a student will raise their hand to get the teacher's attention. A toddler will cry to get his parent's attention.

\section{Behave because It Just Feels} Good!

Sometimes we behave just because that behavior makes us feel good.

For example, a student may fidget with the pen when he is bored at class. A child may such their thumb when they're alone. A boy likes to smell flowers because it smells good.

"Height." Navigate to the location where you want the file to be stored, and click "Export." Use the video editing software of your choice to combine the picture and the audio. For example, if you are using the Photos app on your PC, first launch the app and select the "Video Editor" tab at the top. Click "New video project," and type in the name for the file (e.g., L1S1 for Lesson 1, Slide 1). Click OK to continue. Click the "+ Add" button, and select "From this PC." Select the picture of the slide, and click "Open." Locate the corresponding audio file, and write down how long the audio lasts. Right-click on the picture in the storyboard, and select "Duration." Change the duration to match the length of the audio. Click "Change" to save the setting. Click "Custom audio" at the upper right-hand corner, and select "Add audio file." Select the corresponding audio recording, and click "Open." Click on the audio file you just added, and move the orange-colored bar on the screen so you cut out the 3-s blank recording at the beginning and the end. You can click the triangle-shaped button to test whether the audio is working properly. Once you are done, click "Done." Click "Finish video" in the upper right-hand corner, and select "High" for the video quality. Click "Export," and select the location where you want the file to be saved. Click "Export," and the computer will begin rendering the video. Once the system finishes processing, you will see the file in that location. Repeat the previous steps until all pictures and audio files are combined. Other software that can be used includes Adobe Premiere, iMovie, and Final Cut Pro. Please keep in mind that these steps were performed using PowerPoint 


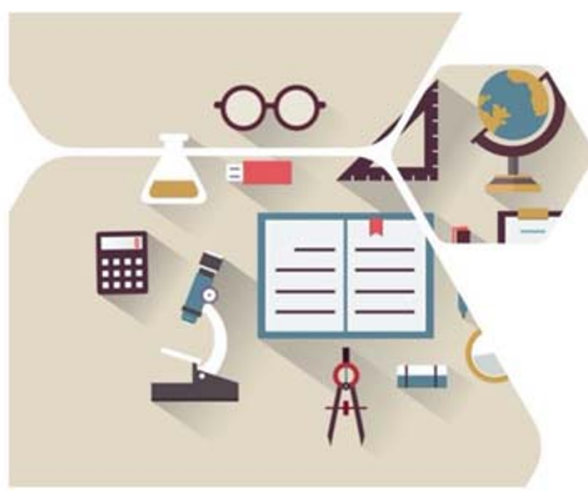

\section{What is}

Behavior?

Lesson 1

\section{Steps to Complete Activity} Schedule

1. Have the child find the first item on the schedule.
2. Have the child complete the activity.

3. The child will place a picture or write down when they are done with the activity.

- It is important for the child to show they are "all done" with the activity. For example, they can cross outcheck off that
activity, or place the activity picture when they are finished. - When learning to use an activity schedule, your child may. need you to remind them to place the checkmark of picture. 5. Repeat all steps until the schedule is complete.

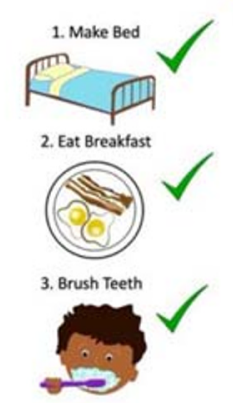

Cashing Out the Tokens: Prize Boxes

- Save up tokens for the chance to pick something from the prize box!
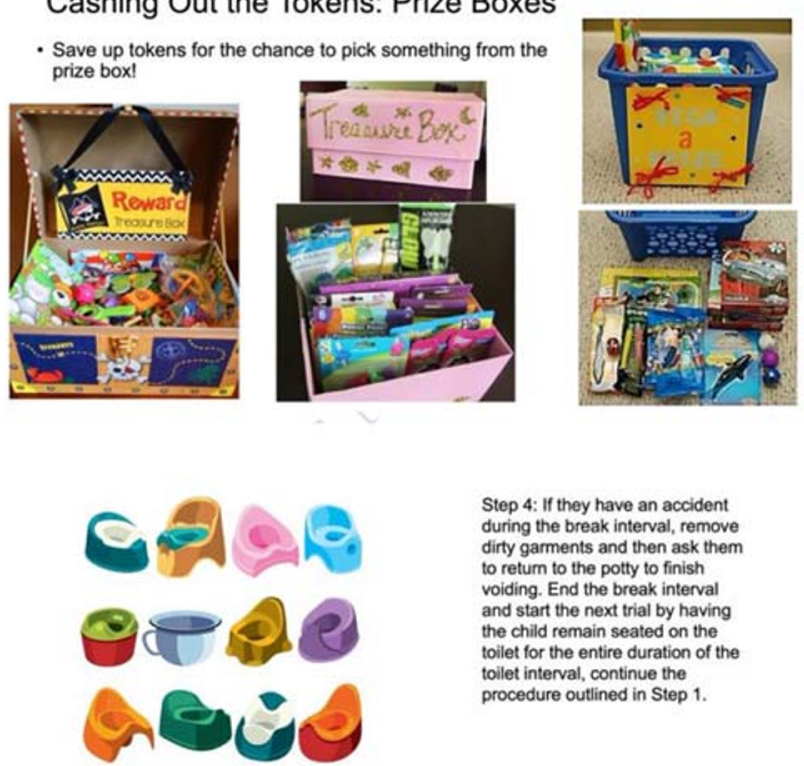

Step 4: If they have an accident during the break interval, remove dirty garments and then ask them to retum to the potty to finish voiding. End the break interval and start the next trial by having the child remain seated on the toilet for the entire duration of the toilet interval, continue the procedure outlined in Step 1.

Steps of the Program
Determine the Function of Behavior

One thing to keep in mind is that the activity you just completed can only identify potential functions of bohaviors. In order to pinpoint its exact function, behavior analysts utilize a process called functional analysis. You should always consult with a protessional before intervening on a persistont and dangerous maladaptive behaviot.
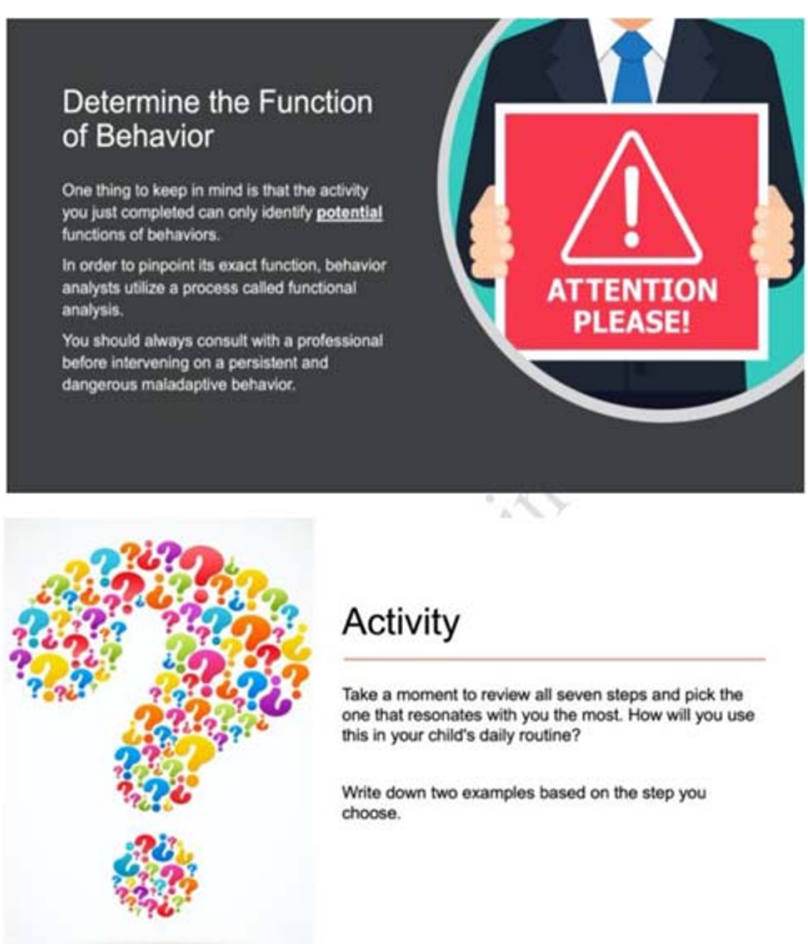

Activity

Take a moment to review all seven steps and pick the one that resonates with you the most. How will you use this in your child's daily routine?

Write down two examples based on the step you
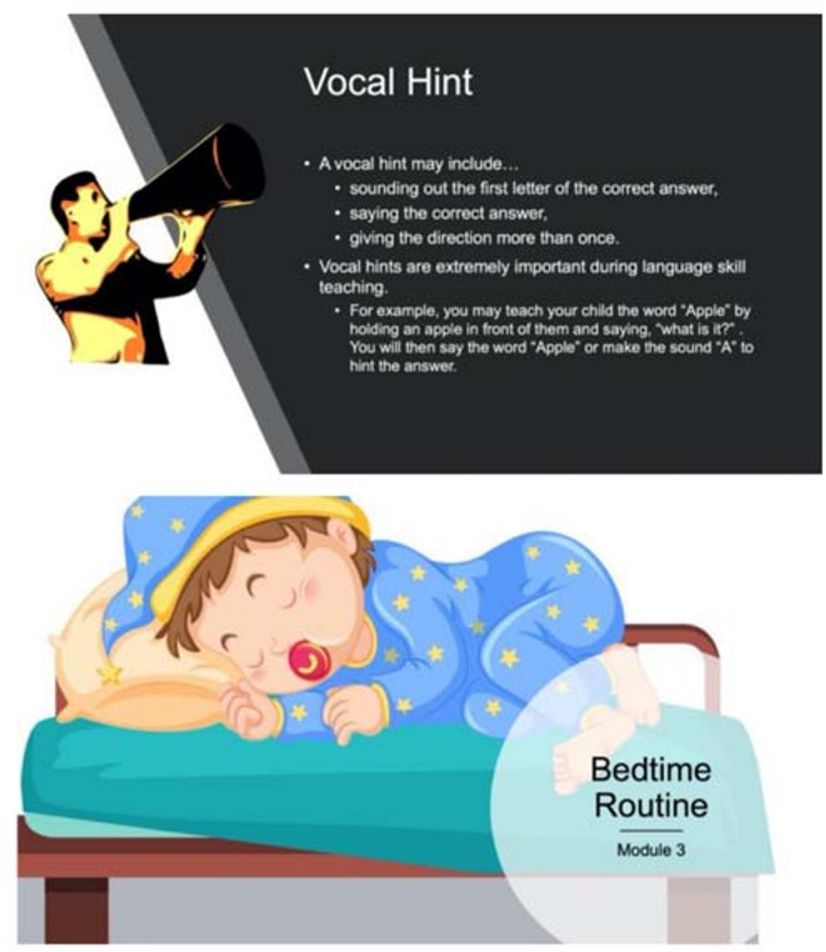

Fig. 4 Examples of finished PowerPoint slides

version 16.37. Older/newer versions of any of this software will likely have minor discrepancies to the steps we outlined previously.
6. Upload course content as videos to the online platform. Once all the videos are ready, upload the video to your online platform (e.g., Moodle, Teachable, Blackboard). 
Depending on the platform, you may enable settings such as requiring parents to watch the entire video before they can move on to the next webpage, requiring the online lessons to be completed in sequential order, logging their progress by showing you what content they have gone through, and logging their percentage of correct responding during knowledge checks. In the example of the current study, a navigation panel is presented to the left of the screen so parents can keep track of their progress. There are four types of webpages: the lecture slide, activities, module knowledge checks, and the Do-ItYourself. On the lecture slide webpage, parents are required to finish watching the video before they can move on to the next part. If they log out before completing the lesson, they will be taken back to where they left off the next time they log in. They can pause and resume the video anytime they want. On the activity webpages, parents are required to pause the video and use the provided workbook to write down their answers. For example, an activity might ask parents to identify potential functions of the behavior in the given scenario. Parents then write down their answers in the blank box. A knowledge check webpage appears at the end of each module. During these checks, multiple-choice and/or true-or-false questions appear one at a time on the screen, and parents are required to complete them before moving on to the next module. Parents receive corrective feedback if they answer the question wrong. A Do-It-Yourself webpage appears at the end of each lesson. The page contains specific instructions for parents to record themselves using one technique covered in the lesson. After completing this activity, parents contact the treatment team and schedule their individual consultations. Figure 5 provides one example for each of these webpages.

\section{Individual Consultations}

As mentioned earlier in the competency-based contingency section, once parents finish one online lesson and complete the required activity, they contact the treatment team and schedule one individual consultation. Each consultation lasts $45 \mathrm{~min}$, and the clinician follows a semistructured checklist to conduct these meetings.

Consultation structure All consultations are conducted via online meeting software (e.g., Zoom, GoToMeeting, Skype). Before each meeting, the clinician reviews parents' progress on the online lessons. If parents make mistakes during the knowledge check, that topic will be discussed in greater detail. Each session begins with a brief review of the online lesson content. The clinician asks parents to share their activity answers, and can clarify any misunderstandings. The clinician also discusses parents' behavioral concerns in relation to the material in the online lessons. For example, the parent learns about the definition of behavior and its
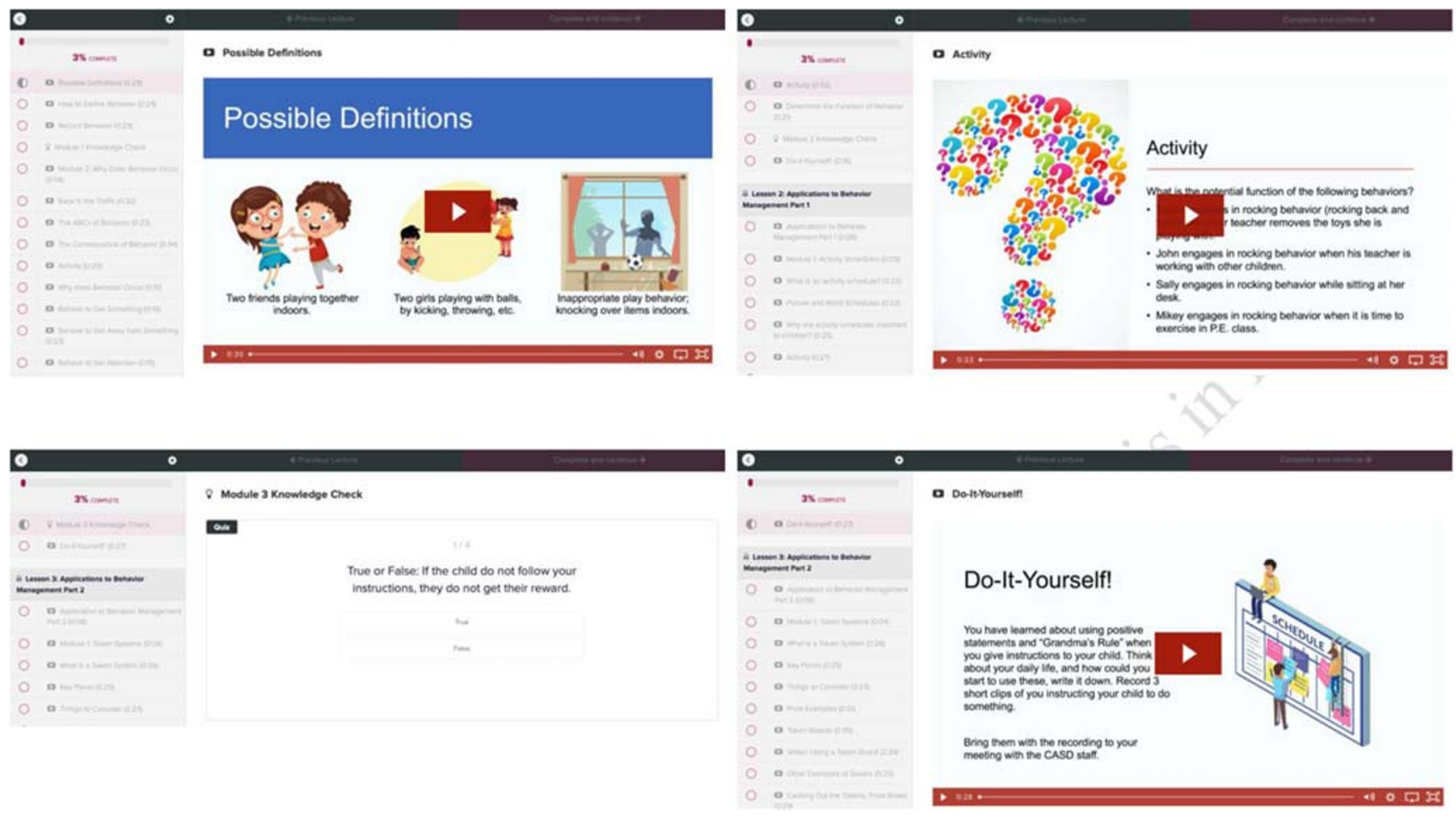

Fig. 5 Examples of four types of webpages in the online lesson 
potential functions. During the discussion, the clinician asks the parent to describe the child's maladaptive behavior. If the parent gives a subjective description, the clinician re-presents the course content and guides the parent to develop a definition that is objective. The clinician also discusses the potential functions of behavior with the parent. This serves two purposes. On the one hand, parents deepen their understanding of the course content. On the other hand, it informs the clinician of potential intervention plans. As parents progress through the program, the content of each consultation changes accordingly. Table 3 presents an outline of how behavioral concerns are discussed in relation to the course content.

Note that Table 3 should be used as a reference. If parents have specific behavioral concerns or if the maladaptive behavior is complex, the clinician should focus more on coaching parents as to how to use specific techniques to address those concerns. The clinician uses a checklist during each consultation. See Figure 6 for an example.

Behavior management plan and coaching During the fifth consultation, the clinician presents a behavior management plan that parents can use in their homes. The focus of the plan will vary depending on the complexity and severity of the maladaptive behavior, as outlined previously in the risk analysis section. These behavior plans should be brief and use non-behavior-analytic terms. Clinicians should prioritize techniques parents already learned throughout the program. The clinician explains the behavior management plan to parents and provides video modeling. After the fifth meeting, parents receive weekly coaching from the clinician. During these coaching sessions, the clinician follows up on the child's progress, answers parents' questions, and makes modifications to the behavior management plan.

\section{Progress Monitoring}

Weekly ACT message Parents receive weekly text messages from the clinic. Besides reminding them to continue the online lesson, the message also briefly describes an ACT activity. These messages aim to increase parents' present moment awareness and promote value-driven behavior. For example, the text message could read as follows:

$\mathrm{Hi}$, this is [name of the agency]. Just a reminder that once you've finished the next online lesson, you can reach out to us to schedule your individual consultation. Additionally, enjoy some mindful moments this weekend! When we think of being mindful, we may think of sitting in a calm, quiet room with no distractions, but that's not always how life is! Sometimes you are in a big crowd or a loud place and may feel the need to slow down and find your way back to the present moment. Throughout the week, when things get busy or hectic, try to focus on the present moment by taking a few deep breaths and focusing on what is going on around you.

Session notes Because all sessions are conducted remotely, and parents may meet with different clinicians due to scheduling conflicts, a detailed session note is of great importance. On the session note, clinicians provide detailed descriptions of what content was covered in the meeting and what examples were used to elaborate on behavioral principles. Clinicians also take note of specific questions raised by parents and document the answer they provided during the session. Clinicians also write down items that need to be followed up with parents in the next session. This information can help the clinical team maintain consistency across sessions. Different clinicians can use the same example to explain different behavioral principles. This will also increase rapport with parents, as they would not need to repeat similar information to different
Table 3 Discussion of Behavioral Concerns

\begin{tabular}{|c|c|c|}
\hline Consultation & Topic & Course Content \\
\hline \multirow[t]{4}{*}{1} & Clarify what the concern is; & How to define behavior \\
\hline & The antecedent and consequence of that behavior; & $\begin{array}{l}\text { The ABCs (antecedent, behavior, } \\
\text { consequence) of behavior }\end{array}$ \\
\hline & The behavior's potential function; and & Functions of behavior \\
\hline & Strategies that the parents have tried in the past. & \\
\hline \multirow[t]{2}{*}{2} & Discuss the child's daily routine; and & Activity schedule \\
\hline & If the child is not responding to instructions. & Instructional control \\
\hline 3 & $\begin{array}{l}\text { Discuss what parents can do at home to manage } \\
\text { those behavior challenges. }\end{array}$ & $\begin{array}{l}\text { Token system, deliver instructions } \\
\text { effectively, redirect behavior }\end{array}$ \\
\hline 4 & Discuss how to teach a replacement behavior. & Natural teaching, structured teaching \\
\hline 5 & Walk through the individualized behavior plan. & Special topics as needed \\
\hline
\end{tabular}


Fig. 6 Parent training discussion checklist used during individual consultations

Name of Clinician: Name of Participant: Date:

\section{Parent Training Discussion Checklist: Parent}

Meeting 1- What is Behavior?

\begin{tabular}{|r|r|}
\hline Topic of Discussion & $\begin{array}{l}\text { Was it } \\
\text { Discussed? Y/N }\end{array}$ \\
\hline Discuss concepts around behavior & \\
\hline Context for behavior & \\
\hline Defining behavior & \\
\hline $\begin{array}{r}\text { Functions of behavior: get something, get away from something, get } \\
\text { someone's attention, and makes us feel good }\end{array}$ & \\
\hline Gather information about the client & \\
\hline Problem behaviors & \\
\hline Pffective strategies the parents have used & \\
\hline Potential reinforcers for client & \\
\hline Review activities & \\
\hline Review knowledge check & \\
\hline Provide simple take-away points before ending & \\
\hline
\end{tabular}

clinicians. Figure 7 provides an example of the session note template.

Briefing, debriefing, and supervision In the 60-day telehealth ABA parent training curriculum described in this example, most clinicians are senior master's-level graduate students enrolled in the Behavior Analysis and Therapy Program. The credential and level of competency needed for clinicians providing consultations should be individualized depending on the depth and complexity of the case. Each family is assigned one case manager and can meet with any member of the treatment team based on availability. Before each meeting, the clinician is required to review all previous session notes and attend a 10-min briefing with the case manager. The case manager reviews the family's information and provides specific directions on what the clinician needs to focus on in the meeting. After the meeting, the clinician is required to debrief the case manager on what happened during the meeting. The case manager is in charge of developing the behavior management plan under the supervision of a BCBA. The BCBA holds a weekly group supervision meeting and also meets with case managers individually as needed.

\section{Conclusion}

Advice on service delivery via telehealth is increasingly prevalent during the current pandemic (CASP, 2020; Rodriguez, 2020). As a science rooted in objective measurable outcomes, it is critical that the advice we give is also effective in producing the outcomes we are expecting. Furthermore, the rise of ACT-related additions (Murrell \& Scherbarth, 2006; Pahnke, Lundgren, Hursti, \& Hirvikoski, 2014) to the typical behavioral training approaches for employees (Bethay, Wilson, Schnetzer, Nassar, \& Bordieri, 2013), clients (Enoch \& Dixon, 2017, 2019), and parents (Blackledge \& Hayes,
2006; Poddar, Sinha, \& Urbi, 2015) necessitates even more proof of concept before inferring such material is warranted for inclusion in enhancing telehealth outcomes.

The previous content details the model of our telehealth ABA parent training curriculum. It includes five self-paced online lessons and details individual consultations parents receive as they move through the program. The content structure covers the majority of behavioral principles and techniques parents need in order to set up the home for the child's success. The individual consultations complement the online lessons. They deepen parents' understanding of the course content and allow behavior analysts to have a detailed discussion on parents' concerns. The brief ACT session during the onboarding meeting, along with the weekly ACT messages, frequently reorients parents to the present moment and enhances parental adherence. Detailed examples are provided so practitioners can replicate this model. Study 2 presents the preliminary data that support the effectiveness of this model. Limitations and suggestions for improvement are also discussed.

\section{Study 2: Evaluating the Yi and Dixon On-Demand Telehealth Model}

\section{Participants and Settings}

Fourteen families from the surrounding geographic region of southern Illinois participated in the 60-day telehealth ABA parent training curriculum described previously. One family was excluded from all data analysis as they did not provide consent for research. Participants were recruited through referral and via the clinic's website. All families had only one child. The average age of children was 8 (range 3-16, SD = 4.00, 11 males and 2 females). All children had a diagnosis of autism. Participants 
Fig. 7 Example of session note template

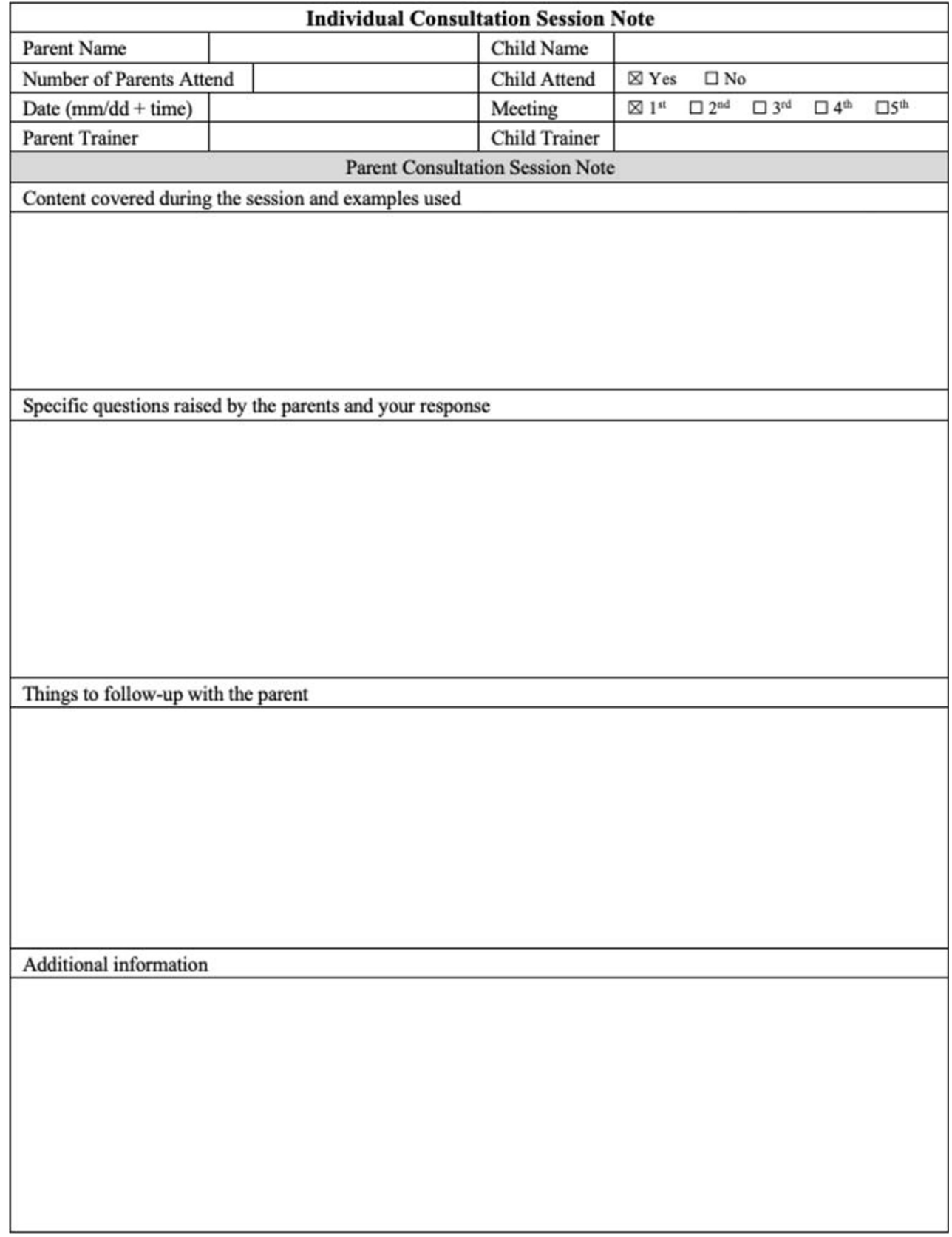

enrolled in the program in early February 2020, which was 1 month before the COVID-19 pandemic began.

\section{Design and Procedures}

The current study used a randomized controlled trial design. Seven families were randomly placed into the ACT group, and six families were placed in the control group. The ACT group participated in the program, as described in Study 1. The control group completed the same activity with modifications during the onboarding meeting and progress monitoring.

During the onboarding meeting, the clinician split families into two groups after providing an overview of the program.
Those in the ACT group remained in the same room and completed the brief 30-min ACT session. Those in the control group were taken to a different room, where the clinician asked parents to discuss the following three topics: what was the most stressful moment they had encountered in the past in relation to their child's challenges, what was the biggest obstacle in the way of the life their child wanted to live, and what was the main goal for them for participating in this program. The amount of time both groups spent with the clinician during this activity was balanced. Once both groups finished this activity, the clinician helped them sign up for the online lessons, and the onboarding meeting ended. When this study began, no stay-at-home orders were currently in place, and a 
telehealth solution for delivery of this content was not required. However, a simple alternative would be to have scheduled a time for each group to participate in this onboarding through the use of Zoom (2020), GoToMeeting (2020), or another form of remote meeting software.

During progress monitoring, parents in the control group received weekly messages simply to remind them to continue the program. Parents in the ACT group received these messages, but with additional text intended to increase their present moment awareness and value-driven behavior. For example, parents in the ACT group received this text message:

$\mathrm{Hi}$, this is [agency name]. Just a reminder that once you've finished the next online lesson, you can reach out to us to schedule your individual consultation. This week, put some space between yourself and your thoughts. Our thoughts are simply words that provoke different feelings, memories, and anticipations of the future. You do not belong to your thoughts. Your thoughts belong to you. You have the power to change the way you interact with your thoughts. This week, anytime you have a thought that causes difficult feelings, repeat the thought out loud in a funny voice. Notice how simply changing the tone can make a difference.

In comparison, parents in the control group received this text message: "Hi, this is [agency name]. Just a reminder that once you've finished the next online lesson, you can reach out to us to schedule your individual consultation."

All other procedures between both groups were the same as described in Study 1.

\section{Program Modification Due to COVID-19}

Before the COVID-19 stay-at-home restrictions, all families were offered the opportunity to conduct their individual consultations in person. In early March, the university where the clinic is housed issued its policy on restricting on-campus activities in response to the COVID-19 outbreak. As a result, all families were offered two options. They could either postpone their participation in the program until the clinic resumed providing in-person consultation, or they could continue participating in the program and receive all future consultations online.

\section{Dependent Measures and Analysis}

Measures were taken to directly evaluate families' progress in the program and also to compare outcomes between the ACT group and the control group. Families' progress during the online lessons was measured once they indicated whether they would continue or postpone participation. All families responded between the 31st and 34th day since enrollment. Specifically, we measured the percentage of online lessons parents had completed, the average number of slides parents went through each time they logged in to the online platform, and their average scores during all completed module knowledge checks. Parents' decisions on whether to continue or postpone the program were analyzed to identify patterns between these two groups. A 5-point Likert-type social validity questionnaire was sent out to families who continued participation 3 weeks after the program modification. The social validity questionnaire assessed the following five components: the relevancy of topics covered in the program, the friendliness of terms and language used in the program, the convenience of the program, the utility of individual consultations, and whether parents could apply what they learned in their homes. The questionnaire was distributed via an online survey platform. All statistical analyses were conducted using the SPSS version 26.0.0.0.

\section{Results}

After the program modification due to COVID-19, $100 \%$ of the families in the control group postponed their participation in the program $(N=6$ of 6$)$. This was considerably higher than the $28 \%$ of families in the ACT group who decided to postpone participation $(N=2$ of 7$)$. Measures on program outcome reported here were taken before this change, which occurred between the 31 st and the 34 th day since parents enrolled in the curriculum.

On average, families in the control group completed $20.5 \%$ of the online lessons $(N=6, S D=31.43 \%)$. They logged in to the platform 2.83 times and each time finished 4.17 slides $(N=$ $6, S D=5.56)$. On average, they scored $93.64 \%$ during all knowledge checks $(N=2, S D=1.93 \%)$. For families in the ACT group, on average, they completed $64.29 \%$ of the online lessons $(N=7, S D=35.00 \%)$. They logged in to the platform 4.43 times and each time finished 16.29 slides $(N=7, S D=$ 11.90). On average, they scored $90.24 \%$ during all knowledge checks $(N=7, S D=2.48 \%)$. Once again, five families in the ACT group decided to continue the program. All families in the control group decided to postpone their participation.

An independent sample $t$ test was conducted to compare the differences in parents' progress. Results indicated a significant difference between parents in the control group and parents in the ACT group. Specifically, parents in the ACT group progressed significantly further in the program than those in the control group, $t(11)=2.36, p=.038$. Parents in the ACT group also finished more slides each time they logged in to the online platform, $t(11)=2.28, p=.044$. The difference in their performance during knowledge checks was not significant, $t(7)=1.75, p=.123$. This result indicated that parents in the ACT group were able to make more progress in the program and that they went through more content each time they 

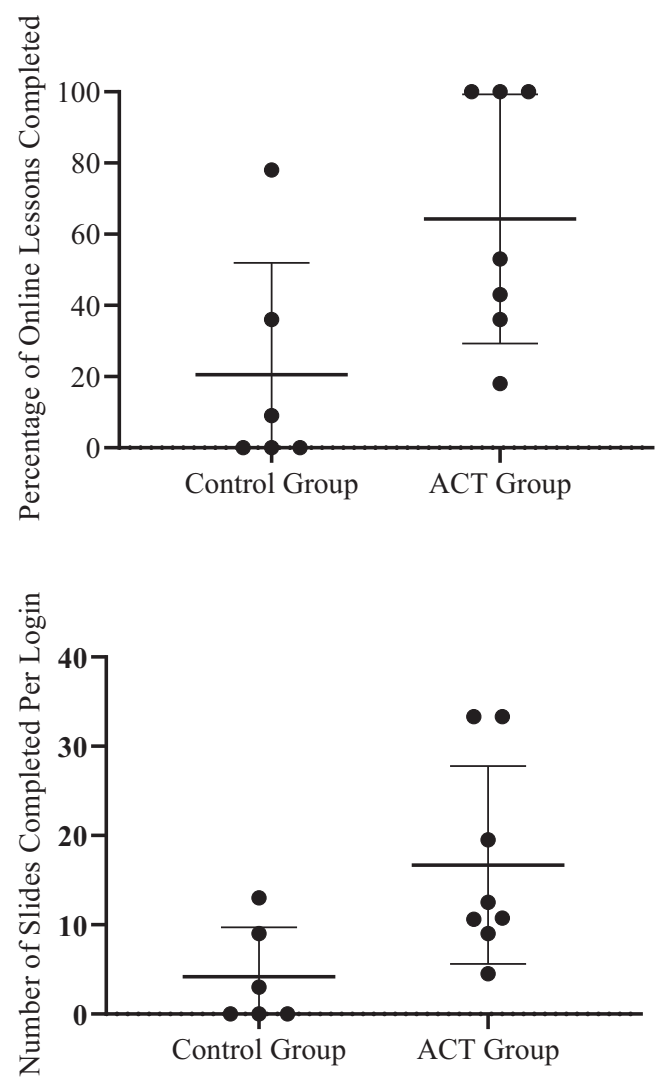

Fig. 8 Differences in the percentage of online lessons completed and the number of slides completed per login between the control group and the ACT group

logged in to the learning platform. However, parents in both groups were able to maintain the knowledge covered in online lessons at the same level, as there was no significant difference in their knowledge check scores. Figure 8 shows the difference in program progress between the two groups.

A chi-square test of association was conducted to identify whether parents in both groups tended to make different decisions during the COVID-19 outbreak. Results indicated a significant difference between these two groups, $\chi^{2}=6.96, p=$ .008 , Cramér's $\mathrm{V}=.732$. This indicated that parents in the ACT group were significantly more likely to continue their participation in the program, whereas parents in the control

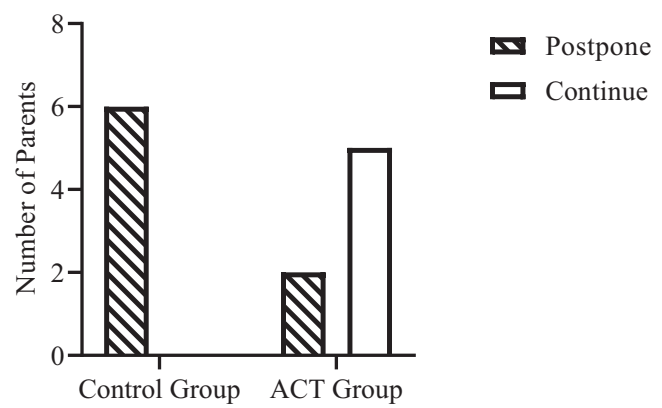

Fig. 9 Number of parents choosing to postpone versus continue the program in the control group and the ACT group group were significantly more likely to postpone their participation. Figure 9 shows the bar graph highlighting this difference between the two groups.

Results from the social validity questionnaire showed that parents rated the program favorably. They indicated that the topics covered in the program were overall relevant to them. The language and examples used made the content less technical and helped them understand behavior-analytic principles and techniques. The self-paced online lessons were very convenient for them. Individual consultations successfully addressed their concerns and questions. Overall, parents indicated that they could use what they learned at home.

\section{Discussion}

Our reported initiatives in on-demand telehealth parent training provide both a detailed guide on how to create such a parent training curriculum and preliminary evidence supporting the effectiveness of its different components to increase parental adherence, such as using non-behavior-analytic terms, incorporating a brief ACT session, and including a competence-based contingency. The current study is in line with previous research that suggests the utility of using a telehealth model in ABA services (Suess, Wacker, Schwartz, Lustig, \& Detrick, 2016; Tomlinson et al., 2018). It extends the study by Vismara, McCormick, Young, Nadhan, and Monlux (2013), demonstrating the effectiveness of an ABA parent training program via remote consultation and self-guided learning platform. The current study addresses the ever-increasing need for evidencebased practices focusing on providing behavior-analytic services remotely and highlights simple techniques clinicians can use to improve the program outcome. The proposed model offers a high level of flexibility so clinicians can address parents' individual needs without significantly modifying the program. This would allow for rapid adoption and decrease the time spent on staff training beforehand, as the overall structure of the program remains the same across families. The selfpaced online lessons allow parents to learn behavioral principles in their own time, which allows the clinician to focus more on addressing each parent's unique needs. The activities and the Do-It-Yourself portion during online lessons guide parents through developing behavior plans by themselves. The videos they record allow the clinician to gain more insight into the child's natural environment, which could increase the effectiveness of the behavior plan.

The format of remote services poses unique challenges, as clinicians are less likely to be able to directly observe the behavior of interest and sessions are less structured when compared with in-person programs. To overcome this, most studies published to date used specific software to facilitate synchronous or asynchronous consultations (Fischer, Clark, Askings, \& Lehman, 2017). The technological difficulties associated with 
this format could affect parents' participation. Lee et al. (2015) highlighted several common issues parents could face during ABA telehealth services, including Internet connectivity, hardware, and software problems. With the clinician providing technological coaching during the onboarding meeting and modeling how to use different software and platforms, the current model decreases parents' response effort during the program.

Another potential challenge of using remote ABA services is the unstructured environment. Because parents complete the program in their natural settings, they are more likely to be distracted than if they attended center-based in-person consultations. The current COVID-19 pandemic could also produce additional psychological stress. By including a brief ACT session during the onboarding meeting and the use of weekly ACT messages, the current model proposes a solution to this issue. Numerous studies have supported the utility of incorporating ACT in ABA services. Bethay et al. (2013) investigated the effect of ACT and instruction in ABA. Their results indicated that participants in the ACT group reported significantly less burnout when working with individuals with intellectual disabilities. Blackledge and Hayes (2006) investigated the effect of ACT on parents of children with autism. The results showed a significant decrease in parental stress and anxiety. Gould et al. (2018) reported a significant increase in overt value-derived behavior among parents with children diagnosed with autism following six sessions of ACT. Recently, Hahs, Dixon, and Paliliunas (2019) examined the effect of ACT-based intervention on parents of children with autism using a randomized controlled trial design. Results indicated that the two 2-hr ACT workshops produced significant clinical outcomes on various measures of psychological flexibility and parental stress. The finding of our current investigation adds to the growing body of research demonstrating the effectiveness of incorporating $\mathrm{ACT}$ in parent training. It extends previous research by showing that a brief 30-min ACT session can significantly improve parents' progress in the curriculum when a global pandemic occurred during the middle of their education. The ACT protocol used in the current study focused on increasing present moment awareness, clarifying values, and prompting value-driven behaviors. It is likely that the COVID-19 pandemic and the disruption to their daily routine would increase parents' stress and alter their current priorities regarding their time allocation. Psychological fusion and experiential avoidance could occur as parents spend more time worrying about the future. By engaging in experiential exercises, clinicians redirected parents back to the present moment. Using protocols that clarified their values and prompted value-driven behavior, parents in the ACT group were more likely to reorient themselves to the parent training program and make more significant progress even in the face of the global pandemic that was encroaching upon their lives. Combined with the weekly ACT message, which aimed at maintaining the effect of the brief ACT session, parents in the ACT group achieved more favorable clinical outcomes.
One interesting finding of the current study is that parents in the ACT group were more likely to continue their participation, whereas all parents in the control group postponed the program. The potential reason behind this difference exceeds the scope of the current article, as we aim to provide a technological guideline to establish a telehealth ABA parent training curriculum and enhance parental adherence. Nevertheless, future research should look into this phenomenon and investigate whether it can be replicated. As the study is limited by a small sample size and between-subject variability, this result should be interpreted with caution.

Future research could also collect data on parents' psychological flexibility and individualize the protocol used during the brief ACT session based on those data. Clinicians could select the experiential exercises and metaphors that are most likely to benefit the parent and could frequently reference those terms during the individual consultation. The effects of more individualized ACT interventions could then be analyzed. In terms of the course content, the current study should be used as a reference. Clinicians could individualize the content and assign topics that are more relevant to the parent. Clinicians could also schedule remote observations and collect data on parents' interactions with their child to examine whether parents are correctly implementing the behavioral techniques.

Overall, the current article provides practical guidelines on how to develop a telehealth ABA parent training curriculum. We provided the structure of the curriculum, as well as protocols to enhance parental adherence. We have described the necessary steps to assemble the program content and suggested practices for monitoring parents' progress. The pandemic of COVID-19 poses unique challenges to the delivery of ABA services, and we hope the current article can guide practitioners to develop their own programs based on the evidence we have. The current study only suggests one possible way of developing such a program. Future explorations should include more evidence on identifying best practices in delivering ABA services remotely, identifying specific protocols on enhancing adherence, and analyzing program outcomes based on overt behaviors of the parent and the child.

Funding Portions of this research was funded by the State of Illinois's The Autism Program (TAP) awarded to the second author.

\section{Compliance with Ethical Standards}

Conflict of Interest The authors have no conflicts of interest to declare that are relevant to the content of this article.

Ethical Approval The content and procedure of this study was approved by the Human Subjects Committee at the Southern Illinois University Carbondale (Date: 01/17/2020, Protocol: 20015).

Consent to Participate Written informed consent was obtained from all participants. 


\section{References}

Ahles, A., \& Jenkins, J. (2018). When the going gets tough, the tough get mindful: A toolkit based on the principles of acceptance and commitment therapy. Retrieved from https://www.helpwithact.com/wpcontent/uploads/clinicians.pdf

Allen, K. D., \& Warzak, W. J. (2000). The problem of parental nonadherence in clinical behavior analysis: Effective treatment is not enough. Journal of Applied Behavior Analysis, 33(3), 373-391.

Becirevic, A., Critchfield, T. S., \& Reed, D. D. (2016). On the social acceptability of behavior-analytic terms: Crowdsourced comparisons of lay and technical language. The Behavior Analyst, 39(2), 305-317.

Behavior Analyst Certification Board. (2020). BACB COVID-19 Updates. Retrieved from https://www.bacb.com/bacb-covid-19updates/

Bethay, J. S., Wilson, K. G., Schnetzer, L. W., Nassar, S. L., \& Bordieri, M. J. (2013). A controlled pilot evaluation of acceptance and commitment training for intellectual disability staff. Mindfulness, 4(2), $113-121$.

Blackledge, J. T., \& Hayes, S. C. (2006). Using acceptance and commitment training in the support of parents of children diagnosed with autism. Child \& Family Behavior Therapy, 28(1), 1-18.

Bondy, A., Horton, C., \& Frost, L. (2020). Promoting functional communication within the home. Behavior Analysis in Practice, 13 (2), 321-328.

Brodhead, M. T. (2015). Maintaining professional relationships in an interdisciplinary setting: Strategies for navigating nonbehavioral treatment recommendations for individuals with autism. Behavior Analysis in Practice, 8(1), 70-78.

Centers for Disease Control and Prevention. (2020). Cases in U.S. Retrieved from https://www.cdc.gov/coronavirus/2019-ncov/casesupdates/cases-in-us.html\#weekly-surveillance-report

Cox, D. J., Plavnick, J. B., \& Brodhead, M. T. (2020). A proposed process for risk mitigation during the COVID-19 pandemic. Behavior Analysis in Practice, 13(2), 299-305.

Desire2Learn [Computer software] (2020). Kitchener, Ontario, Canada: D2L Corporation. Retrieved from http://www.d21.com

Enoch, M. R., \& Dixon, M. R. (2017). The use of a child-based acceptance and commitment therapy curriculum to increase attention. Child \& Family Behavior Therapy, 39(3), 200-224.

Enoch, M. R., \& Dixon, M. R. (2019). Neuro-typical children outcomes from an acceptance and commitment therapy summer camp. Behavior Analysis in Practice, 12(2), 343-352.

Fischer, A. J., Dart, E. H., Leblanc, H., Hartman, K. L., Steeves, R. O., \& Gresham, F. M. (2016). An investigation of the acceptability of videoconferencing within a school-based behavioral consultation framework. Psychology in the Schools, 53(3), 240-252.

Fischer, A. J., Clark, R., Askings, D., \& Lehman, E. (2017). Technology and telehealth applications. In J. K. Luiselli (Ed.), Applied behavior analysis advanced guidebook (pp. 135-163). San Diego, CA, United States: Academic Press.

GoToMeeting [Computer software]. (2020). Boston, MA: LogMeIn Inc. Retrieved from http://www.gotomeeting.com

Gould, E. R., Tarbox, J., \& Coyne, L. (2018). Evaluating the effects of acceptance and commitment training on the overt behavior of parents of children with autism. Journal of Contextual Behavioral Science, 7, 81-88.

Hahs, A. D., Dixon, M. R., \& Paliliunas, D. (2019). Randomized controlled trial of a brief acceptance and commitment training for parents of individuals diagnosed with autism spectrum disorders. Journal of Contextual Behavioral Science, 12, 154-159.

Hay-Hansson, A. W., \& Eldevik, S. (2013). Training discrete trials teaching skills using videoconference. Research in Autism Spectrum Disorders, 7(11), 1300-1309.
Higgins, W. J., Luczynski, K. C., Carroll, R. A., Fisher, W. W., \& Mudford, O. C. (2017). Evaluation of a telehealth training package to remotely train staff to conduct a preference assessment. Journal of Applied Behavior Analysis, 50(2), 238-251.

Lee, J. F., Schieltz, K. M., Suess, A. N., Wacker, D. P., Romani, P. W., Lindgren, S. D., . . Dalmau, Y. C. P. (2015). Guidelines for developing telehealth services and troubleshooting problems with telehealth technology when coaching parents to conduct functional analyses and functional communication training in their homes. Behavior Analysis in Practice, 8(2), 190-200.

Lindgren, S., Wacker, D., Suess, A., Schieltz, K., Pelzel, K., Kopelman, T., et al. (2016). Telehealth and autism: Treating challenging behavior at lower cost. Pediatrics, 137(Supp. 2), S167-S175.

Loeber, R., \& Weisman, R. (1975). Contingencies of therapist and trainer performance: A review. Psychological Bulletin, 82(5), 660.

McIntyre, L. L., Gresham, F. M., DiGennaro, F. D., \& Reed, D. D. (2007). Treatment integrity of school-based interventions with children. Journal of Applied Behavior Analysis, 40(4), 659-672.

Moodle [Computer software]. (2020). West Perth, WA, Australia: The Moodle Project. Retrieved from http://moodle.org

Murrell, A. R., \& Scherbarth, A. J. (2006). State of the research \& literature address: ACT with children, adolescents and parents. International Journal of Behavioral Consultation and Therapy, 2(4), 531.

Pahnke, J., Lundgren, T., Hursti, T., \& Hirvikoski, T. (2014). Outcomes of an acceptance and commitment therapy-based skills training group for students with high-functioning autism spectrum disorder: A quasi-experimental pilot study. Autism, 18(8), 953-964.

Poddar, S., Sinha, V., \& Urbi, M. (2015). Acceptance and commitment therapy on parents of children and adolescents with autism spectrum disorders. International Journal of Educational and Psychological Researches, 1(3), 221.

Reinert, K. S., Higbee, T. S., \& Nix, L. D. (2020). Creating digital activity schedules to promote independence and engagement. Behavior Analysis in Practice, 13(3), 577-595.

Rodriguez, K. A. (2020). Maintaining treatment integrity in the face of crises: A treatment selection model for transitioning direct ABA services to telehealth. Behavior Analysis in Practice, 13(2), 291298.

Schramm, R., \& Miller, M. (2014). The seven steps to earning instructional control. Morrisville, NC, United States: Lulu Press.

Suess, A. N., Wacker, D. P., Schwartz, J. E., Lustig, N., \& Detrick, J. (2016). Preliminary evidence on the use of telehealth in an outpatient behavior clinic. Journal of Applied Behavior Analysis, 49(3), 686-692.

Teachable [Computer software]. (2020). New York, NY: Teachable Inc. Retrieved from http://teachable.com

The Council of Autism Service Providers. (2020). Practice parameters for telehealth $A B A$. Retrieved from https://casproviders.org/wpcontent/uploads/2020/03/PracticeParametersTelehealthABA 040320.pdf

Tomlinson, S. R., Gore, N., \& McGill, P. (2018). Training individuals to implement applied behavior analytic procedures via telehealth: A systematic review of the literature. Journal of Behavioral Education, 27(2), 172-222.

Vismara, L. A., McCormick, C., Young, G. S., Nadhan, A., \& Monlux, K. (2013). Preliminary findings of a telehealth approach to parent training in autism. Journal of Autism and Developmental Disorders, 43(12), 2953-2969.

Zoom [Computer software]. (2020). San Jose, CA: Zoom Video Communications Inc. Retrieved from http://zoom.us

Publisher's Note Springer Nature remains neutral with regard to jurisdictional claims in published maps and institutional affiliations. 